\title{
Sarcopenia and frailty: new challenges for clinical practice
}

\author{
Authors: Richard Dodds ${ }^{\mathrm{A}}$ and Avan Aihie Sayer ${ }^{\mathrm{B}}$
}

Sarcopenia and frailty are important conditions that become increasingly prevalent with age. Sarcopenia is the loss of muscle mass and function, and frailty can be defined as multi-system impairment associated with increased vulnerability to stressors. There is overlap between the two conditions, especially in terms of the physical aspects of the frailty phenotype: low grip strength, gait speed and muscle mass. These measures have been associated with a wide range of ageing outcomes and can be assessed in the clinical setting. In terms of intervention, there is evidence for the benefit of resistance exercise programmes, although these may not always be feasible. Considerable research into the use of medicines, both existing and new, as well as dietary supplements is ongoing. Finally in order to prevent or delay the development of these conditions, an additional approach is to consider aetiological factors operating across the life course.

KEYWORDS: Sarcopenia, frailty, muscle function, muscle mass, ageing, life course

\section{Introduction}

The term sarcopenia, from the Greek meaning loss of flesh, was first suggested in 1989, with more recent definitions incorporating the loss of muscle function as well as the loss of muscle mass that occurs with ageing. ${ }^{1}$ It is a common and increasingly important condition as populations grow older and it is associated with subsequent disability and morbidity. Sarcopenia is also associated with substantial financial cost: the healthcare costs of sarcopenia in the USA in 2000 were

Authors: ${ }^{A}$ Wellcome Trust research training fellow and specialty registrar in geriatric medicine, Academic Geriatric Medicine, MRC Lifecourse Epidemiology Unit, University of Southampton, Southampton UK; ${ }^{\text {B }}$ professor of geriatric medicine and MRC programme leader, Academic Geriatric Medicine, MRC Lifecourse Epidemiology Unit, University of Southampton, Southampton UK; NIHR Southampton Biomedical Research Centre, University of Southampton and University Hospital Southampton NHS Foundation Trust, Southampton UK; NIHR CLAHRC Wessex, University of Southampton, Southampton, UK; Newcastle University Institute of Ageing and Institute of Health and Society, Newcastle University, Newcastle upon Tyne, UK estimated to be $\$ 18.5$ billion. $^{2}$ However, perhaps the most striking indication of the importance of sarcopenia comes from the evidence linking poor muscle function, in particular weak grip strength, to increased all-cause mortality rates in middleaged and older people. ${ }^{3}$ Frailty can be defined as multi-system impairment associated with increased vulnerability to stressors. ${ }^{4}$ There is considerable overlap between the two conditions, especially in terms of the physical aspects of frailty. The aim of this review is to summarise how research is being translated to the diagnosis and treatment of sarcopenia and frailty.

\section{Diagnosis and relevant outcomes}

Diagnostic criteria are clearly essential for the recognition of sarcopenia and frailty in clinical practice. The algorithm for sarcopenia published by the European Working Group on Sarcopenia in Older People (EWGSOP) in 2010 requires the presence of either low gait speed or low grip strength to then test for low muscle mass as shown in Fig 1. There is considerable overlap with the classic frailty phenotype definition by Fried et $a l^{5}$ which also includes low gait speed and low grip strength, as well as weight loss (as a proxy for loss of muscle mass), selfreported low physical activity and exhaustion. The presence of three or more of these five features indicates frailty. Another approach is to use a frailty index, which is the proportion of a number of deficits, such as hearing impairment, that are present in a pre-specified list of 30 or more items covering

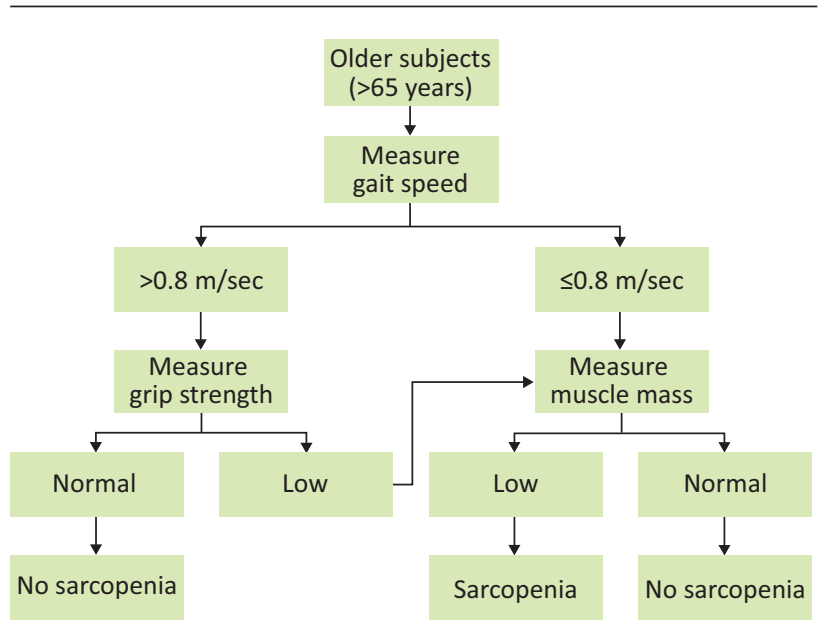

Fig 1. The algorithm suggested by the European Working Group on Sarcopenia in Older People for diagnosing sarcopenia. Reproduced with permission. ${ }^{1}$ 
multiple systems. ${ }^{4}$ This approach requires more information to be collected, although it does allow the degree of frailty to be assessed. This section will now focus on the objective measures that are common to the EWGSOP definition of sarcopenia and Fried frailty, namely gait speed, muscle strength and muscle mass. How can these characteristics be measured in a clinical setting and what do they tell us about an older person's current and future health?

\section{Gait speed}

Older people with slow gait speed have been found to be at an increased risk of subsequent disability, falls, cognitive decline, institutionalisation and mortality. ${ }^{6}$ Gait speed is readily assessed in the clinical setting by measuring the time taken to walk a set distance, such as $4 \mathrm{~m}$, at usual pace. Although there appears to be a continuous relationship between gait speed and outcomes such as mortality, ${ }^{6}$ for clinical purposes a range of cut-points have been proposed, such as $0.8 \mathrm{~m} / \mathrm{sec}$, as used in the EWGSOP definition for sarcopenia.

\section{Grip strength}

Grip strength has been recommended as the most practical method of measuring muscle strength in the clinical setting ${ }^{1}$ and has been found to correlate physical performance measures in the lower limbs. ${ }^{7}$ The Jamar dynamometer is the most commonly described device. ${ }^{8}$ In a systematic review, 7 out of 10 studies of older people found that weak grip predicted either incident disability or worsening of existing disability. ${ }^{9}$ Weaker grip strength has also been associated with increased length of hospital stay. ${ }^{10}$ There is strong evidence linking grip strength with mortality rates, with a meta-analysis of 14 studies showing a graded relationship between weaker grip and increased risk of death; ${ }^{3}$ the hazard ratio comparing the lowest to the highest quarters of grip strength was 1.67 (95\% confidence interval 1.45-1.93). Whereas associations between measures of physical performance and mortality have been assessed mainly in older populations, four of the studies included in the grip strength meta-analysis had an average age at baseline of below 60 years, and the findings here were similar. Normative data are available for grip ${ }^{11,12}$ and cut points have been proposed, including those from the FNIH Sarcopenia Project which found that cut-points of $26 \mathrm{~kg}$ in men and $16 \mathrm{~kg}$ in women best identified individuals with mobility disability (assessed using slow gait speed) at ages 65 and older. ${ }^{13}$

\section{Muscle mass}

Possible techniques for measuring muscle mass in the clinical setting include anthropometry, bioelectrical impedance (BIA) and dual energy X-ray absorptiometry (DXA). Anthropometric measures are prone to error and are not considered to be suitable for assessing muscle mass in older people. ${ }^{1}$ BIA, which produces estimates of total fat mass and lean mass, has the advantage over DXA that the equipment used is portable. However it has been questioned to what extent BIA provides additional information beyond that from anthropometric measurements (weight and height) alone. The third technique, DXA, can accurately estimate the proportion of lean tissue, fat tissue and bone, although access to scanning equipment may be a limiting factor. Baumgartner et $\mathrm{al}^{14}$ proposed an index of relative skeletal muscle mass, in the form of appendicular skeletal muscle mass $(\mathrm{kg})$ from DXA divided by height $(\mathrm{m})$ squared. They also suggested cut-offs for sarcopenia in the form of two standard deviations below the gender-specific young adult mean: $7.26 \mathrm{~kg} / \mathrm{m}^{2}$ for men and 5.45 $\mathrm{kg} / \mathrm{m}^{2}$ for women. There is evidence that strength may be more predictive of the risk of subsequent disability and mortality than muscle mass. ${ }^{15}$ There is also debate around the feasibility of measuring muscle mass in the potentially large numbers of older people who may fall below thresholds proposed for physical performance and strength. ${ }^{16}$

\section{Prevalence of sarcopenia and frailty}

Patel et $a l^{17}$ examined the prevalence of sarcopenia using the EWGSOP definition in the Hertfordshire Cohort Study: at mean age 67 years, $4.6 \%$ of men and $7.9 \%$ of women were found to have sarcopenia. Gale et al ${ }^{18}$ found a similar prevalence of Fried frailty in the English Longitudinal Study of Ageing: $6.5 \%$ of those aged $60-69$ years. Sarcopenia and frailty both increase markedly with age; for example, Gale et al found that $65 \%$ of those over 90 years old met the Fried criteria for frailty.

\section{Treatment}

There are a range of potential treatments for sarcopenia and frailty. Most current evidence has used measures such as muscle strength and gait speed as outcomes; going forward, the optimal measures to use as outcomes in trials on sarcopenia and frailty is an important issue. This section describes the established role of exercise programmes, along with the potential to modify diet and drug treatments. There is also observational evidence that factors across the life course may affect an individual's likelihood of developing sarcopenia and frailty, as shown in Fig 2; this exciting potential area for intervention is considered at the end of this section.

\section{Exercise}

Resistance exercise is the most studied form of exercise intervention. The 2009 Cochrane review of the use of progressive resistance training to improve physical function ${ }^{19}$ included studies with an average age of at least 60 years old, the majority of which were high-intensity programmes performed

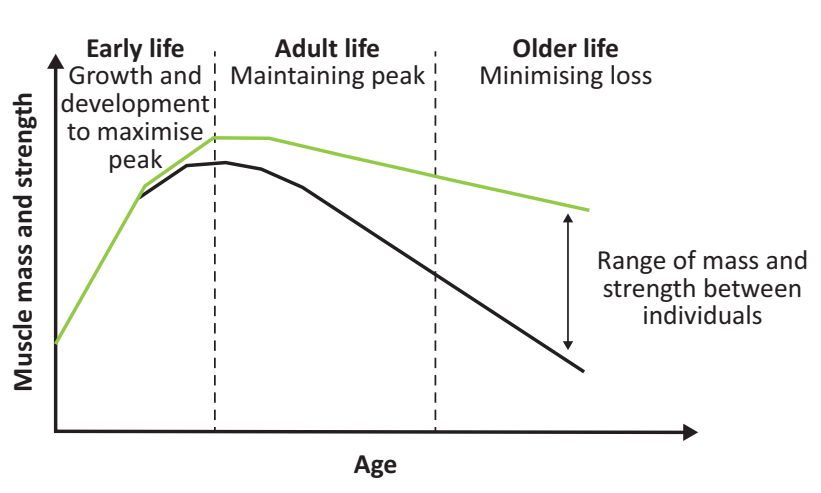

Fig 2. The life course approach. Reproduced with permission. ${ }^{36}$ 
twice or three times per week in gym or clinic-based settings. The outcomes used in different studies varied but there was evidence of a moderate-to-large beneficial effect on strength in the lower limb, as well as a moderate effect on gait speed. There is also evidence that resistance exercise is beneficial for lean mass, with a pooled finding across 49 studies of an increase in lean mass of $1.1 \mathrm{~kg}$ following an average of 20.5 weeks of resistance exercise. ${ }^{20}$ As with physical function, the benefits were seen especially in high-intensity programmes. Other types of exercise intervention include aerobic exercise, balance and flexibility training and functional training. These have been less studied in regard to outcomes related to sarcopenia.

\section{Diet}

Less is known about dietary interventions than the established role of resistance training. There is considerable recent literature which suggests that several aspects of diet may be important in the development of sarcopenia. ${ }^{21}$ Food intake falls by approximately $25 \%$ from 40 to 70 years of age, and particularly if combined with a tendency towards a monotonic diet, may lead to inadequate nutrient intake. Three key areas have been considered with respect to diet in sarcopenia: protein, vitamin D and antioxidants.

Protein provides the amino acids required for muscle synthesis. There is also evidence that the amino acid leucine may activate the signalling pathways leading to protein synthesis. A trial in relatively young (mean age 71) and healthy men failed to show on effect on muscle mass or strength, however, perhaps because the group studied tended to have diets already replete in leucine. ${ }^{22}$ There is also a general concern that the muscle synthesis in older people following a protein load may be blunted, leading to the suggestion that recommended overall protein intakes for older people should be increased to $1.2-1.5 \mathrm{~g} / \mathrm{kg} / \mathrm{day} .^{23}$ However a Cochrane review found no consistent effect of supplements on functional measures relevant to sarcopenia. ${ }^{24}$ The quantity and composition of dietary protein for the prevention and treatment of sarcopenia therefore remains unclear.

The current widespread interest in diseases potentially related to vitamin-D deficiency includes sarcopenia and frailty. Evidence supporting a role for vitamin D includes the fact that polymorphisms in vitamin $\mathrm{D}$ have been linked to muscle strength, and frailty has been shown to be associated with vitamin-D deficiency. There is consensus that low vitamin-D levels require replacement, ${ }^{23}$ but as with protein supplementation, intervention trials of the effect of vitamin D on strength and physical performance have shown mixed results.

The accumulation of reactive oxygen species (ROS) in older age is recognised to have a role in muscle wasting, although the precise forms of ROS responsible and their interactions are not fully understood. ${ }^{25}$ This in turn makes it difficult to know which specific antioxidants are likely to be of benefit as supplements and there have been few trials. There is evidence from observational studies that those with higher overall antioxidant status have better physical function as well as attenuated decline in measures such as walking speed. ${ }^{26}$

In summary, a common finding across the three types of dietary intervention is a mismatch between the findings from observational and intervention studies. One possible explanation for this is the tendency for intake of dietary components to be highly correlated with one another; hence the association between one marker of a healthy diet and physical function may be confounded by other components. Indeed there is some evidence linking 'healthy' diets, containing wholemeal cereals and greater amounts of fruit and vegetables, to greater muscle strength in older people. ${ }^{21}$ An important area for further research is therefore the potential of whole-diet interventions, which attempt to change dietary patterns rather than focusing on specific nutrients in isolation.

\section{Medication}

As yet there is no recommended drug treatment for sarcopenia or frailty. However sarcopenia is now a major focus for drug discovery. ${ }^{27}$ This follows in part from the fact that although resistance training has been shown to be effective, many older people may be unable or unwilling to exercise at the required intensity. One area which has been explored is hormone administration. Growth hormone has been shown to increase muscle mass but not clearly alter functional outcomes and is therefore of questionable benefit. ${ }^{28}$ This highlights the challenge of choosing outcome measure(s) for trials in sarcopenia. Testosterone supplementation has been found to increase both muscle mass and strength in men but has been linked to adverse cardiovascular events. ${ }^{29}$ A current area of interest is in drugs affecting the renin-angiotensin system, and whether these might have direct effects on muscle. An observational study initially suggested that angiotensin-converting enzyme inhibitors might be of benefit for physical function, a finding subsequently confirmed in a trial showing improved six minute walk time in those given perindopril. ${ }^{30}$ Further trials are ongoing.

\section{A life course approach to sarcopenia and frailty}

A complementary approach to the treatments already described is to consider the aetiological factors for sarcopenia and frailty across the life course. This substantially broadens the window for understanding the aetiology of these conditions and for developing interventions to prevent or delay their development. The life course epidemiology framework views function in old age as the combination of two phases: growth to the peak obtained in early adult life and subsequent decline thereafter, as shown in Fig 2.

The association between birth weight and muscle strength was first described in the Hertfordshire Ageing Study. ${ }^{31}$ This finding has since been replicated, with a systematic review describing a positive association between birth weight and muscle strength in 17 out of 19 studies. ${ }^{32}$ Furthermore, longer duration of breastfeeding has been linked to higher grip strength in men in the Hertfordshire Cohort Study. ${ }^{33}$ Studies have also examined the relationship between lifestyle factors, including physical activity in childhood and adulthood and muscle strength. For example, in the MRC National Survey of Health and Development, a cumulative benefit of increased leisure time physical activity across ages $36,43,53$ and 60-64 has been found for grip strength at age 60-64 years. ${ }^{34}$ Future epidemiological studies will continue to improve our understanding of factors across the life course that may prevent sarcopenia and frailty, including opportunities to examine the cellular and molecular mechanisms that underlie such associations. $^{35}$ 


\section{Conclusions}

In conclusion, this review has covered recent developments in the diagnosis and treatment of sarcopenia and frailty. The development of consensus definitions for sarcopenia has helped to inform clinical assessment of patients as well as recruitment into trials. At present, progressive resistance training is the most well studied intervention, although access, uptake and response are variable. Research into these conditions is expanding exponentially and includes how we might be able to intervene earlier in the life course to prevent their occurrence.

\section{Funding}

RD is supported by a Wellcome Trust fellowship (grant number WT099055AIA).

\section{Note}

This article was originally published in the 2015 Clinical Medicine supplement Horizons in Medicine 27. All articles in this supplement are available at www.clinmed.rcpjournal.org/content/15/Suppl_6

\section{References}

1 Cruz-Jentoft AJ, Baeyens JP, Bauer JM et al. Sarcopenia: European consensus on definition and diagnosis: Report of the European Working Group on Sarcopenia in Older People. Age Ageing 2010;39:412-23.

2 Janssen I, Shepard DS, Katzmarzyk PT, Roubenoff R. The healthcare costs of sarcopenia in the United States. J Am Geriatr Soc 2004;52:80-5.

3 Cooper R, Kuh D, Hardy R. Objectively measured physical capability levels and mortality: systematic review and meta-analysis. BMJ 2010;341:c4467.

4 Clegg A, Young J, Iliffe S et al. Frailty in elderly people. Lancet 2013;381:752-62.

5 Fried LP, Tangen CM, Walston J et al. Frailty in older adults: evidence for a phenotype. J Gerontol A Biol Sci Med Sci 2001;56A:M146-56.

6 Studenski S, Perera S, Patel K et al. Gait speed and survival in older adults. JAMA 2011;305:50-8.

7 Visser M, Deeg DJ, Lips P et al. Skeletal muscle mass and muscle strength in relation to lower-extremity performance in older men and women. J Am Geriatr Soc 2000;48:381-6.

8 Roberts HC, Denison HJ, Martin HJ et al. A review of the measurement of grip strength in clinical and epidemiological studies: towards a standardised approach. Age Ageing 2011;40:423-9.

9 Vermeulen J, Neyens JCL, van Rossum E et al. Predicting ADL disability in community-dwelling elderly people using physical frailty indicators: a systematic review. BMC Geriatr 2011;11:33.

10 Kerr A, Syddall HE, Cooper C et al. Does admission grip strength predict length of stay in hospitalised older patients? Age Ageing 2006;35:82-4.

11 Bohannon RW, Peolsson A, Massy-Westropp N et al. Reference values for adult grip strength measured with a Jamar dynamometer: a descriptive meta-analysis. Physiotherapy 2006;92:11-5.

12 Dodds RM, Syddall HE, Cooper R et al. Grip strength across the life course: normative data from twelve british studies. PLoS One 2014;9:e113637.

13 Alley DE, Shardell MD, Peters KW et al. Grip strength cutpoints for the identification of clinically relevant weakness. J Gerontol A Biol Sci Med Sci 2014;69:559-66.

14 Baumgartner RN, Koehler KM, Gallagher D et al. Epidemiology of sarcopenia among the elderly in New Mexico. Am J Epidemiol 1998;147:755-63.

15 Manini TM, Clark BC. Dynapenia and aging: an update. J Gerontol A Biol Sci Med Sci 2012;67:28-40.
16 Keevil VL, Hayat S, Dalzell N et al. The physical capability of community-based men and women from a British cohort: the European Prospective Investigation into Cancer (EPIC)-Norfolk study. BMC Geriatr 2013;13:93.

17 Patel HP, Syddall HE, Jameson K et al. Prevalence of sarcopenia in community-dwelling older people in the UK using the European Working Group on Sarcopenia in Older People (EWGSOP) definition: findings from the Hertfordshire Cohort Study (HCS). Age Ageing 2013;42:378-84.

18 Gale CR, Cooper C, Sayer A. Prevalence of frailty and disability: findings from the English Longitudinal Study of Ageing. Age Ageing 2014;44:162-5.

19 Liu C, Latham N. Progressive resistance strength training for improving physical function in older adults. Cochrane Database Syst Rev 2009;(3):CD002759.

20 Peterson M, Sen A, Gordon P. Influence of resistance exercise on lean body mass in aging adults: a meta-analysis. Med Sci Sports Exerc 2012;43:249-58.

21 Robinson S, Cooper C, Aihie Sayer A. Nutrition and sarcopenia: a review of the evidence and implications for preventive strategies. J Aging Res 2012;2012:510801.

22 Verhoeven S, Vanschoonbeek K, Verdijk LB et al. Long-term leucine supplementation does not increase muscle mass or strength in healthy elderly men. Am J Clin Nutr 2009;89:1468-75.

23 Morley J, Argiles J, Evans W et al. Nutritional recommendations for the management of sarcopenia. J Am Med Dir Assoc 2010;11:391-6.

24 Milne AC, Potter J, Vivanti A, Avenell A. Protein and energy supplementation in elderly people at risk from malnutrition. Cochrane Database Syst Rev 2009;15:CD003288.

25 Arthur PG, Grounds MD, Shavlakadze T. Oxidative stress as a therapeutic target during muscle wasting: considering the complex interactions. Curr Opin Clin Nutr Metab Care 2008;11:408-16.

26 Kaiser M, Bandinelli S, Lunenfeld B. Frailty and the role of nutrition in older people. A review of the current literature. Acta Biomed 2010;81:37-45.

27 Brass EP, Sietsema KE. Considerations in the development of drugs to treat sarcopenia. J Am Geriatr Soc 2011;59:530-5.

28 Giannoulis MG, Martin FC, Nair KS et al. Hormone replacement therapy and physical function in healthy older men. Time to talk hormones? Endocr Rev 2012;33:314-77.

29 Xu L, Freeman G, Cowling BJ, Schooling CM. Testosterone therapy and cardiovascular events among men: a systematic review and meta-analysis of placebo-controlled randomized trials. BMC Med 2013;11:108.

30 Sumukadas D, Witham Struthers MD McMurdo MET AD. Effect of perindopril on physical function in elderly people with functional impairment: a randomized controlled trial. CMAJ 2007;177:867-74.

31 Aihie Sayer A, Cooper C, Evans JR et al. Are rates of ageing determined in utero? Age Ageing 1998;27:579-83.

32 Dodds R, Denison HJ, Ntani G et al. Birth weight and muscle strength: a systematic review and meta-analysis. J Nutr Heal Ageing 2012;16:609-15.

33 Robinson SM, Simmonds SJ, Jameson KA et al. Muscle strength in older community-dwelling men is related to type of milk feeding in infancy. J Gerontol A Biol Sci Med Sci 2012;67:990-6.

34 Dodds R, Kuh D, Aihie Sayer A, Cooper R. Physical activity levels across adult life and grip strength in early old age : updating findings from a British birth cohort. Age Ageing 2013;42:794-8.

35 Patel HP, Syddall HE, Martin HJ et al. Hertfordshire sarcopenia study: design and methods. BMC Geriatr 2010;10:43.

36 Aihie Sayer A, Syddall H, Martin H et al. The developmental origins of sarcopenia. J Nutr Heal Ageing 2008;12:427-32.

Address for correspondence: Prof AA Sayer, Academic Geriatric Medicine, MRC Lifecourse Epidemiology Unit, University of Southampton, Southampton General Hospital, Southampton S016 6YD, UK.

Email: aas@mrc.soton.ac.uk 